\title{
Optimal Mass Design of 25 Bars Truss with Loading Conditions on Five Node Elements
}

\author{
Koumbe Mbock $^{1 *}$, Etoua Remy Magloire ${ }^{2}$ \\ Department of Mathematics and Physics \\ National Advanced School of Engineering, Yaounde, P.O. Box 8390, Cameroon \\ E-mails: ${ }^{1}$ k.mbock@yahoo.fr, ${ }^{2}$ retoua@yahoo.fr \\ ${ }^{*}$ Corresponding author \\ Lezin Seba Minsili³, Okpwe Mbarga Richard ${ }^{4}$ \\ Department of Civil Engineering \\ National Advanced School of Engineering, Yaounde, P.O. Box 8390, Cameroon \\ ${ }^{3}$ lezinsm@yahoo.com, ${ }^{4}$ okrichar@gmail.com
}

(Received August 14, 2018; Accepted October 18, 2018)

\begin{abstract}
The optimal design of a twenty-five bar space truss commonly involves multiple loading conditions acting on 4 node elements in the linear elastic model. In this paper, we describe the behavior of the truss system with our experimental loading conditions on five node elements subject to minimum displacement and stresses that are used to formulate the constrained nonlinear optimization problem. Numerical computations are developed with the objective of mass minimization and the best structural design is selected by applying the interior point method with the guidance of Matlab Optimization Toolbox. Our numerical results show the optimal values of cross-sectional areas, material densities, and internal forces which satisfy the minimum weight design. These results provide the appropriate mass to the experimental data and allow substantial changes in size, shape, and topology.
\end{abstract}

Keywords- Linear elastic model, Variational problem, Structural optimization, Interior point method, Trusses

\section{Introduction}

The twenty-five bars space truss problem is typically characterized by their large numbers of design variables and constraints within allowable limits. It is therefore difficult but not impossible to describe this truss system with all stresses and displacements for minimum weight, deflection and for the maximum fundamental natural frequency of vibration. This structure has been optimized by many workers including Rajeev and Krishnamoorthy (1992), Wu and Chow (1995a, 1995b), Adeli and Park (1996), Erbatur et al. (2000), Park and Sung (2002). In these studies, the loading conditions were acting on four node elements using the size and shape symmetry in certain study case. Other studies were made with the same loading conditions including Venkayya (1971), Schmit and Farshi (1974), Schmidt and Miura (1976), Adeli and Kamal (1986), Saka (1990), Lamberti (2008), Farshi and Ziazi (2010). To make the structure economical and save materials, the truss profiles were selected for optimal mass design using STADD.Pro in Bora (2016) and also in Jha and Paliwal (2017).

In this paper, we examine the case where the loading conditions are acting on five nodes elements in a linear elastic model in order to select the economical profile of the 25 bars space truss. For this, we find the displacement field from the variational problem and look for the design requirements and structural criteria that leads to the objective of mass minimization. Numerical 
International Journal of Mathematical, Engineering and Management Sciences

Vol. 4, No. 1, 1-16, 2019

https://dx.doi.org/10.33889/IJMEMS.2019.4.1-001

computations are developed about applied forces at five node elements to select the best structural design of minimal mass. By applying the interior point-method (Herskovits et al., 2000) with the guidance of Matlab optimization Toolbox (Coleman et al., 1999), the results provide the economical mass, which allows substantial changes in size, shape, and topology of the truss system.

The purpose is introduced in this section and the linear elastic model is formulated in section 2 with the minimization of the energy functional. Structural analysis is developed about the loading conditions on five nodes elements in order to state the objective of the mass minimization problem in section 3 . The structural design of the minimum mass is selected in section 4 through numerical applications on two load cases and section 5 concludes the present study.

\section{Model Formulation and Minimization of the Energy Functional}

We consider that a twenty-five bars truss shown in Figure 1, occupies a domain $\Omega$ of $\mathrm{N}$ dimensional design space and carries a load on the nodes $\{1\},\{2\},\{3\},\{5\}$ and $\{6\}$ as given below in Figure 2.

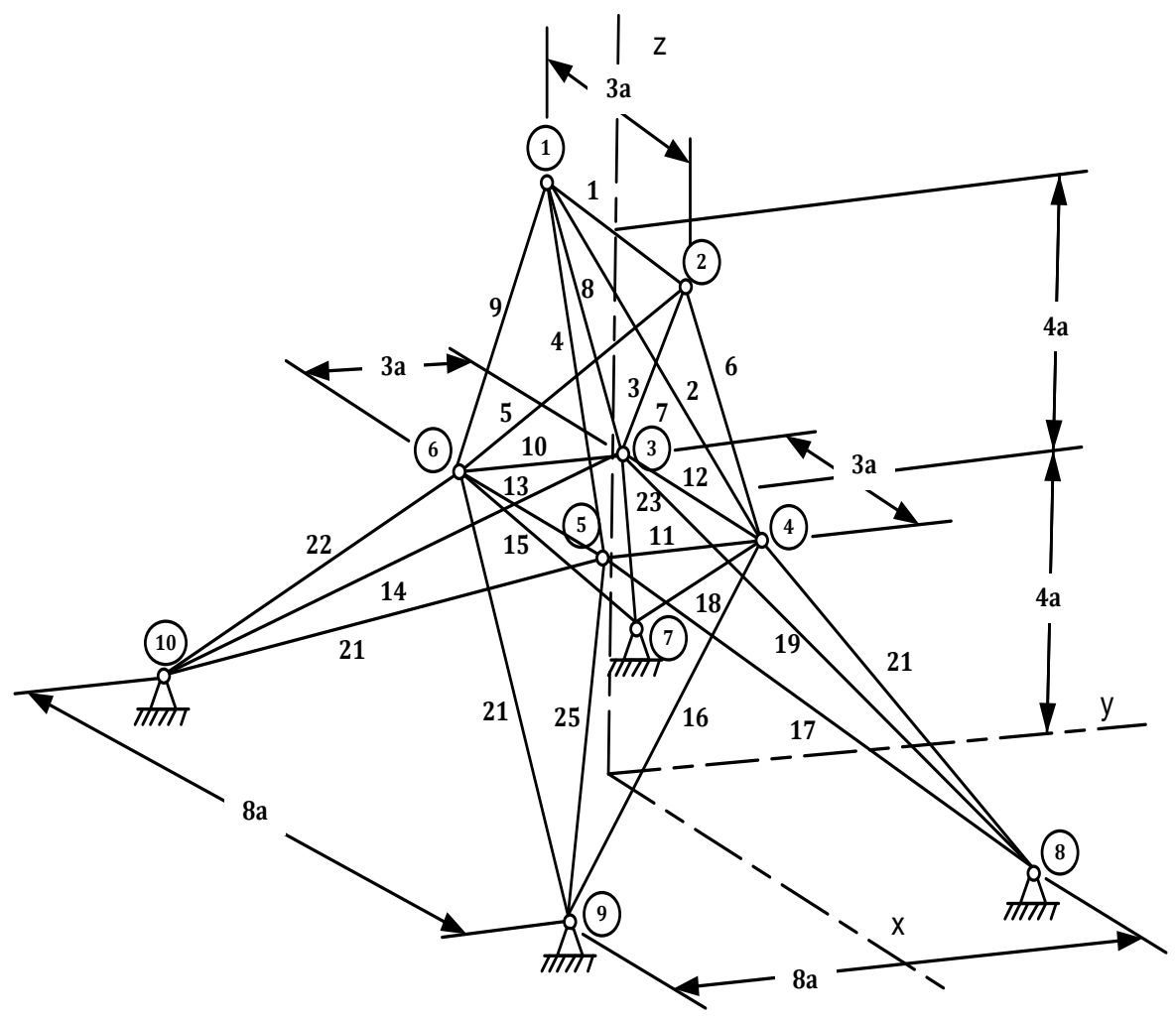

Figure 1. Twenty-five bars truss 
International Journal of Mathematical, Engineering and Management Sciences

Vol. 4, No. 1, 1-16, 2019

https://dx.doi.org/10.33889/IJMEMS.2019.4.1-001

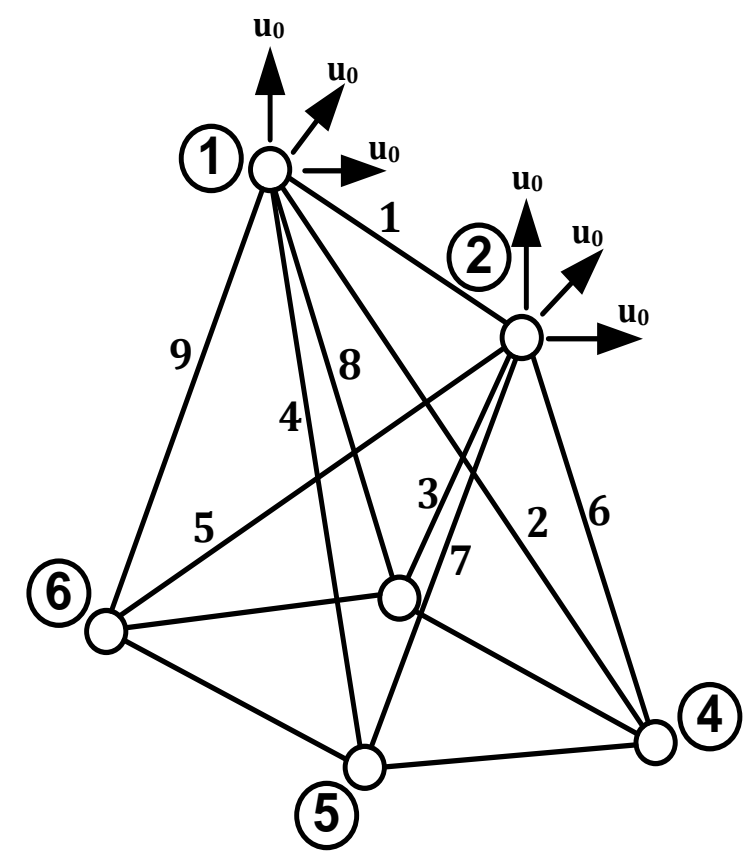

Figure 2. Applied loads at the Nodes $\{1\},\{2\},\{3\},\{5\}$ and $\{6\}$

The structural design of a twenty-five bars truss commonly involves a mathematical model in term of partial differential equations, a set of objective functions and a set of admissible constraints or stresses. In general, one denotes by the Hilbert space $\mathcal{L}^{2}(\Omega)$, the set of measurable and square integrable functions in $\Omega$ and $\mathcal{H}^{1}(\Omega)=\left\{\omega \in \mathcal{L}^{2}(\Omega) \mid \forall \mathrm{i} \in\{1,2, \ldots, \mathrm{N}\}, \frac{\partial \omega}{\partial \mathrm{x}_{\mathrm{i}}} \in \mathcal{L}^{2}(\Omega)\right\}$, the Sobolev space. The boundary of $\Omega$ is denoted by $\partial \Omega$ and the loading forces $F \in \mathcal{L}^{2}(\Omega)$ are imposed on the boundary $\partial \Omega_{\mathrm{F}}=\partial\{\{1\},\{2\},\{3\},\{5\},\{6\}\}$ by $\mathrm{g}_{\mathrm{i}} \in \mathcal{L}^{2}(\Omega)$ withi $\in\{1,2,3,5,6\}$. At the nodes $\{7\},\{8\},\{9\}$ and $\{10\}$, the truss is fixed in contact with the soil and we assume that all the displacements are equal to zero on the boundary denoted by $\partial \Omega_{\mathrm{u}_{0}}=\partial\{\{7\},\{8\},\{9\},\{10\}\}$. In Hilbert space of all displacement $\mathcal{H}^{1}(\Omega)$, we denote by $\mathrm{u}_{\mathrm{ad}}$, the admissible displacements at the nodes $\{1\}$ and $\{2\}$ and on the boundary $\partial \Omega_{\mathrm{u}_{1}}=\partial\{\{1\},\{2\}\}$. The loading conditions are not applied on the boundary $\partial \Omega_{\mathrm{F}_{0}}=\mathrm{C}_{\partial \Omega}^{\partial \Omega_{\mathrm{F}} \cup \partial \Omega_{\mathrm{u}}}$ and the overall boundary can be decomposed in such a manner that $\partial \Omega=\partial \Omega_{\mathrm{F}} \cup \partial \Omega_{\mathrm{u}_{0}} \cup \partial \Omega_{\mathrm{u}_{1}} \cup \partial \Omega_{\mathrm{F}_{0}}$. We consider that $\mathrm{f} \in \mathcal{L}^{2}(\Omega)$ is the volume force of the truss structure and assume that the elastic properties of the cross sections are represented by a symmetric matrix function $\mathrm{A}(\mathrm{x})=\left(\mathrm{a}_{\mathrm{i}, \mathrm{j}}(\mathrm{x})\right)_{1 \leq \mathrm{i}, \mathrm{j} \leq \mathrm{N}} \in \mathrm{IR}^{\mathrm{N} \times \mathrm{N}}$. According to Hooke law, the normal component of the stresses field $\sigma=\sum_{i=1}^{N}\left(\sum_{j=1}^{N} \frac{\partial \sigma_{i, j}}{\partial x_{j}}\right) \in \mathcal{L}^{2}(\Omega)^{N}$ is equal to $A \nabla u$ and the elastic model equation is represented by 
International Journal of Mathematical, Engineering and Management Sciences

Vol. 4, No. 1, 1-16, 2019

https://dx.doi.org/10.33889/IJMEMS.2019.4.1-001

$\operatorname{div} \sigma=\mathrm{f}$, in $\Omega$

$\mathrm{u}=0$, on $\partial \Omega_{\mathrm{u}_{0}}=\partial\{\{7\},\{8\},\{9\},\{10\}\}$

$\mathrm{u}=\mathrm{u}_{\mathrm{ad}}$, on $\partial \Omega_{\mathrm{u}_{1}}=\partial\{\{1\},\{2\}\}$

$\mathrm{F}=\sigma . \mathrm{n}=\mathrm{g}_{\mathrm{i}}$, on $\partial \Omega_{\mathrm{F}}=\partial\{\{1\},\{2\},\{3\},\{5\},\{6\}\}$

$\mathrm{F}=\sigma . \mathrm{n}=0$, on $\partial \Omega_{\mathrm{F}_{0}}$

The solution space to model (1-5) is $\mathcal{H}_{\mathrm{s}}(\Omega)=\left\{\mathrm{u} \in \mathcal{H}^{1}(\Omega) \mid \mathrm{u}=0\right.$ on $\partial \Omega_{\mathrm{u}_{0}}$ and $\mathrm{u}=$ $\mathrm{u}_{\mathrm{ad}}$ on $\left.\partial \Omega_{\mathrm{u}_{1}}\right\}$ and the variational problem can be stated as:

$\int_{\Omega} \mathrm{A} \nabla \mathrm{u} . \nabla \mathrm{vdx}=\int_{\Omega} \mathrm{f} . \mathrm{vdx}+\int_{\partial \Omega_{\mathrm{F}}} \mathrm{g}_{\mathrm{i}} \cdot \mathrm{vds}$,

for all $v \in \mathcal{H}_{\mathrm{s}}(\Omega)$, with $\mathrm{i} \in\{1,2,3,5,6\}$.

The Dirichlet conditions (2) and (3) are satisfied in the solution space $\mathcal{H}_{\mathrm{S}}(\Omega)$ and the Neumann conditions (4) and (5) are imposed in the variational formulation. We assume that the unique solution of the variational problem (6) is also the unique solution of the boundary value problem (1-5). Its stability depends continuously on the surface force $g_{i} \in \mathcal{L}^{2}(\Omega)$ and the volume force $f \in$ $\mathcal{L}^{2}(\Omega)$. This solution is obtained by minimizing the energy functional $\mathrm{J}(\mathrm{v})=\frac{1}{2} \mathrm{q}(\mathrm{v})-$ $\left(\int_{\Omega} \mathrm{f} . \mathrm{vdx}+\int_{\partial \Omega_{\mathrm{F}}} \mathrm{g}_{\mathrm{i}} \cdot \mathrm{vds}\right)$ in the Hilbert space $\mathcal{H}_{\mathrm{s}}(\Omega)$ as follows

$\mathrm{u}=\arg \min _{\mathrm{v} \in \mathcal{H}_{\mathrm{s}}(\Omega)} \mathrm{J}(\mathrm{v})$

where the bilinear form $\int_{\Omega} \mathrm{A} \nabla \mathrm{u} . \nabla \mathrm{vdx} \in \mathcal{H}_{\mathrm{s}}(\Omega)$ can admit a quadratic form denoted by $\mathrm{q}(\mathrm{v})$.

The problem (7) provides the minimum displacement $\mathrm{u} \in \mathcal{H}_{\mathrm{s}}(\Omega)$ which is used to attain the objective of the mass minimization.

\subsection{Stress and Displacement Constraints of Our Approach}

One denotes by $\mathrm{U}_{\mathrm{ad}} \subset \mathcal{H}_{\mathrm{s}}(\Omega)$ and $\sigma_{\mathrm{ad}} \subset \mathcal{H}_{\mathrm{s}}(\Omega)$, the set of admissible displacements and stresses in tension or compression. Also, we assume that the coordinates at each node of the twenty-five bars truss are given in Table 1.

Table 1. Coordinate systems with respect to $\mathrm{x}, \mathrm{y}$, and $\mathrm{z}$-axis

\begin{tabular}{|c|c|c|c|}
\hline Nodes & $\mathbf{x}$ & $\mathbf{Y}$ & $\mathbf{Z}$ \\
\hline 1 & $-3 / 2$ & 0 & 8 \\
\hline 2 & $3 / 2$ & 0 & 8 \\
\hline 3 & $-3 / 2$ & $3 / 2$ & 4 \\
\hline 4 & $3 / 2$ & $3 / 2$ & 4 \\
\hline 5 & $3 / 2$ & $-3 / 2$ & 4 \\
\hline 6 & $-3 / 2$ & $-3 / 2$ & 0 \\
\hline 7 & -4 & 4 & 0 \\
\hline 8 & 4 & 4 & 0 \\
\hline 5 & 4 & -4 & 4 \\
\hline 10 & $3 / 2$ & $-3 / 2$ & 0 \\
\hline
\end{tabular}


International Journal of Mathematical, Engineering and Management Sciences

Vol. 4, No. 1, 1-16, 2019

https://dx.doi.org/10.33889/IJMEMS.2019.4.1-001

As it is shown in Table 2, one denotes by $\left(\mathrm{l}_{\mathrm{i}}\right)_{1 \leq i \leq 25}>0$, the ith length or line joining two node elements and by $\left(\mathrm{S}_{\mathrm{i}, \mathrm{j}}\right)_{1 \leq \mathrm{i}, \mathrm{j} \leq 25} \in \Omega$, the members of cross-sections. The formula used to compute the lengths are given by: $\mathrm{l}_{\mathrm{i}}=\sqrt{\Delta \mathrm{x}_{\mathrm{i}}^{2}+\Delta \mathrm{y}_{\mathrm{i}}^{2}+\Delta \mathrm{z}_{\mathrm{i}}^{2}}$.

Table 2. Length $\left(l_{i}\right)_{1 \leq i \leq 25}$ associated to the cross sections $\left(S_{i, j}\right)_{1 \leq i, j \leq 25, i \neq j}$

\begin{tabular}{|c|c|}
\hline Cross sections & Lengths \\
\hline $\mathrm{S}_{1, \mathrm{j}}$ & 3 \\
\hline$S_{2, j}$ & $\sqrt{109} / 2$ \\
\hline$S_{3, j}$ & $\sqrt{109} / 2$ \\
\hline $\mathrm{S}_{4, \mathrm{j}}$ & $\sqrt{109} / 2$ \\
\hline$S_{5, j}$ & $\sqrt{109} / 2$ \\
\hline$S_{6, j}$ & $\sqrt{73} / 2$ \\
\hline$S_{8, j}$ & $\sqrt{73} / 2$ \\
\hline$S_{9, j}$ & $\sqrt{73} / 2$ \\
\hline$S_{10, j}$ & 3 \\
\hline$S_{11, j}$ & 3 \\
\hline$S_{12, j}$ & 3 \\
\hline$S_{13, j}$ & 3 \\
\hline$S_{14, j}$ & $\sqrt{210} / 2$ \\
\hline $\mathrm{S}_{15, \mathrm{j}}$ & $\sqrt{210} / 2$ \\
\hline$S_{16, j}$ & $\sqrt{210} / 2$ \\
\hline $\mathrm{S}_{17, \mathrm{j}}$ & $\sqrt{210} / 2$ \\
\hline $\mathrm{S}_{18, \mathrm{j}}$ & $\sqrt{210} / 2$ \\
\hline $\mathrm{S}_{19, \mathrm{j}}$ & $\sqrt{210} / 2$ \\
\hline $\mathrm{S}_{20, \mathrm{j}}$ & $\sqrt{210} / 2$ \\
\hline$S_{21, j}$ & $\sqrt{210} / 2$ \\
\hline$S_{22, j}$ & $\sqrt{114} / 2$ \\
\hline$S_{23, j}$ & $\sqrt{114} / 2$ \\
\hline$S_{24, j}$ & $\sqrt{114} / 2$ \\
\hline $\mathrm{S}_{25, \mathrm{j}}$ & $\sqrt{114} / 2$ \\
\hline
\end{tabular}

We assume the permissible displacements on the node element 1 and 2 as it is presented in Table 3.

Table 3. Displacement limit $u_{0}$ at the nodes $\{1\}$ and $\{2\}$

\begin{tabular}{|c|c|c|c|}
\hline Nodes & x-direction & y-direction & z-direction \\
\hline 1 & \pm 3.5 & \pm 3.5 & \pm 3.5 \\
\hline 2 & \pm 3.5 & \pm 3.5 & \pm 3.5 \\
\hline
\end{tabular}

Each node has three degrees of freedom with respect to $\mathrm{x}, \mathrm{y}$ and $\mathrm{z}$-axis and these constraints of displacement on the nodes 1 and 2 are given by the equations: 
International Journal of Mathematical, Engineering and Management Sciences

Vol. 4, No. 1, 1-16, 2019

https://dx.doi.org/10.33889/IJMEMS.2019.4.1-001

$\left|u_{1 x}\right| \leq 3.5=u_{0}, \quad\left|u_{1 y}\right| \leq 3.5=u_{0}, \quad\left|u_{1 z}\right| \leq 3.5=u_{0}$
$\left|u_{2 x}\right| \leq 3.5=u_{0}, \quad\left|u_{2 y}\right| \leq 3.5=u_{0}, \quad\left|u_{2 z}\right| \leq 3.5=u_{0}$

The displacements imposed on the nodes 1 and 2 influence other nodes of the truss system. It appears an axial deformation at each member of the cross sections modeled by the displacements. We have the relation between axial deformations and stresses by $\sigma_{0}=\mathrm{E} \varepsilon$ where $\mathrm{E}$ is the modulus of elasticity and $\varepsilon=\frac{\Delta \mathrm{l}}{\mathrm{l}}$. Therefore we can consider the limitation of stresses presented in Table 4 .

Table 4. Description of the elastic behavior

\begin{tabular}{|c|c|}
\hline Cross sections & Stresses \\
\hline $\mathrm{S}_{1, \mathrm{j}}$ & $\frac{2}{3} \mathrm{Eu}_{0}$ \\
\hline $\mathrm{S}_{2, \mathrm{j},}, \mathrm{S}_{3, \mathrm{j},}, \mathrm{S}_{4, \mathrm{j}, \mathrm{S}} \mathrm{S}_{5, \mathrm{j}}$ & $\frac{10}{109} \mathrm{Eu}_{0}$ \\
\hline $\mathrm{S}_{6, \mathrm{j}}, \mathrm{S}_{7, \mathrm{j}}, \mathrm{S}_{8, \mathrm{j},}, \mathrm{S}_{9, \mathrm{j}}$ & $\frac{26}{73} \mathrm{Eu}_{0}$ \\
\hline
\end{tabular}

We denote by $h=\left\{h_{i, j} \in \mathcal{H}^{1}(\Omega) \mid 1 \leq \mathrm{i} \leq 2\right.$ and $1 \leq \mathrm{j} \leq 6$ and $\left.\mathrm{i} \neq \mathrm{j}\right\}$, the set of internal forces that are distributed in $\Omega_{\mathrm{F}}$ from the imposed displacements on the nodes 1 and 2 . The behavior law leads to the following constraints:

$\left|\mathrm{h}_{1,2}\right| \leq \frac{2}{3} \mathrm{Eu}_{0}, \quad\left|\mathrm{~h}_{1,4}\right| \leq \frac{10}{209} \mathrm{Eu}_{0}, \quad\left|\mathrm{~h}_{2,3}\right| \leq \frac{10}{209} \mathrm{Eu}_{0}$

$\left|\mathrm{h}_{1,5}\right| \leq \frac{10}{209} \mathrm{Eu}_{0}, \quad\left|\mathrm{~h}_{2,6}\right| \leq \frac{10}{209} \mathrm{Eu}_{0}, \quad\left|\mathrm{~h}_{1,6}\right| \leq \frac{10}{209} \mathrm{Eu}_{0}$

$\left|\mathrm{h}_{1,3}\right| \leq \frac{26}{73} \mathrm{Eu}_{0}, \quad\left|\mathrm{~h}_{2,4}\right| \leq \frac{26}{73} \mathrm{Eu}_{0}, \quad\left|\mathrm{~h}_{2,5}\right| \leq \frac{26}{73} \mathrm{Eu}_{0}$

The admissible constraint field $\sigma_{0}$ has to be in tension or in compression and those stresses are represented in $\Omega$ by the following equations:

$\left|\mathrm{h}_{1,2}\right| \leq \sigma_{0} \mathrm{~S}_{1,2}, \quad\left|\mathrm{~h}_{1,3}\right| \leq \sigma_{0} \mathrm{~S}_{1,3}, \quad\left|\mathrm{~h}_{1,4}\right| \leq \sigma_{0} \mathrm{~S}_{1,4}$

$\left|\mathrm{h}_{1,2}\right| \leq \sigma_{0} \mathrm{~S}_{1,2}, \quad\left|\mathrm{~h}_{1,3}\right| \leq \sigma_{0} \mathrm{~S}_{1,3}, \quad\left|\mathrm{~h}_{1,4}\right| \leq \sigma_{0} \mathrm{~S}_{1,4}$

$\left|h_{1,4}\right| \leq \sigma_{0} S_{1,4}, \quad\left|h_{1,5}\right| \leq \sigma_{0} S_{1,5}, \quad\left|h_{1,6}\right| \leq \sigma_{0} S_{1,6}$

$\left|\mathrm{h}_{2,3}\right| \leq \sigma_{0} \mathrm{~S}_{2,3}, \quad\left|\mathrm{~h}_{2,4}\right| \leq \sigma_{0} \mathrm{~S}_{2,4}, \quad\left|\mathrm{~h}_{2,5}\right| \leq \sigma_{0} \mathrm{~S}_{2,5}$

$\left|h_{2,6}\right| \leq \sigma_{0} S_{2,6}, \quad\left|h_{3,4}\right| \leq \sigma_{0} S_{3,4}, \quad\left|h_{3,6}\right| \leq \sigma_{0} S_{3,6}$

$\left|h_{3,7}\right| \leq \sigma_{0} S_{3,7}, \quad\left|h_{3,8}\right| \leq \sigma_{0} S_{3,8}, \quad\left|h_{3,10}\right| \leq \sigma_{0} S_{3,10}$

$\left|\mathrm{h}_{4,5}\right| \leq \sigma_{0} \mathrm{~S}_{4,5}, \quad\left|\mathrm{~h}_{4,8}\right| \leq \sigma_{0} \mathrm{~S}_{4,8}, \quad\left|\mathrm{~h}_{5,6}\right| \leq \sigma_{0} \mathrm{~S}_{5,6}$

$\left|h_{5,8}\right| \leq \sigma_{0} S_{5,8}, \quad\left|h_{5,9}\right| \leq \sigma_{0} S_{5,9}, \quad\left|h_{5,10}\right| \leq \sigma_{0} S_{5,10}$

$\left|h_{6,7}\right| \leq \sigma_{0} S_{6,7}, \quad\left|h_{6,9}\right| \leq \sigma_{0} S_{6,9}, \quad\left|h_{6,10}\right| \leq \sigma_{0} S_{6,10}$

$\left|h_{6,10}\right| \leq \sigma_{0} S_{6,10}$ 
International Journal of Mathematical, Engineering and Management Sciences

Vol. 4, No. 1, 1-16, 2019

https://dx.doi.org/10.33889/IJMEMS.2019.4.1-001

\subsection{External Stability Conditions of the Truss System}

For stability, we denoted by $\mathrm{F}=\left\{\mathrm{F}_{\mathrm{i}, \mathrm{j}} \in \mathcal{H}^{1}(\Omega) \mid 1 \leq \mathrm{i} \leq 6\right.$ and $\left.1 \leq \mathrm{j} \leq 10\right\}$ the set of external forces $\mathrm{F}_{\mathrm{i}, \mathrm{j}}$ which satisfies the equilibrium state of 25 bars space truss. The projections are represented in $\Omega$ on the node elements with respect to $\mathrm{x}, \mathrm{y}$ and $\mathrm{z}$ axis and the equations describing this equilibrium are given as follows:

Projections on the node 1:

$$
\begin{aligned}
& \mathrm{h}_{1,2}+\frac{6}{\sqrt{109}} \mathrm{~h}_{2,3}+\frac{6}{\sqrt{109}} \mathrm{~h}_{1,5}=\mathrm{F}_{1, \mathrm{x}} \\
& \frac{3}{\sqrt{73}} \mathrm{~h}_{1,3}+\frac{3}{\sqrt{109}} \mathrm{~h}_{1,4}-\frac{3}{\sqrt{109}} \mathrm{~h}_{1,5}-\frac{3}{73} \mathrm{~h}_{1,6}=\mathrm{F}_{1, \mathrm{y}} \\
& -\frac{3}{\sqrt{73}} \mathrm{~h}_{1,3}-\frac{8}{\sqrt{109}} \mathrm{~h}_{1,4}-\frac{8}{\sqrt{109}} \mathrm{~h}_{1,5}-\frac{8}{73} \mathrm{~h}_{1,6}=\mathrm{F}_{1, \mathrm{z}}
\end{aligned}
$$

Projections on the node 2:

$$
\begin{aligned}
& \mathrm{h}_{1,2}-\frac{6}{\sqrt{109}} \mathrm{~h}_{2,3}-\frac{6}{\sqrt{109}} \mathrm{~h}_{2,6}=\mathrm{F}_{2, \mathrm{x}} \\
& \frac{3}{\sqrt{109}} \mathrm{~h}_{2,3}+\frac{3}{\sqrt{73}} \mathrm{~h}_{2,4}-\frac{3}{\sqrt{73}} \mathrm{~h}_{2,5}-\frac{3}{109} \mathrm{~h}_{2,6}=\mathrm{F}_{2, \mathrm{y}} \\
& -\frac{8}{\sqrt{109}} \mathrm{~h}_{2,3}-\frac{8}{\sqrt{73}} \mathrm{~h}_{2,4}+\frac{8}{\sqrt{73}} \mathrm{~h}_{2,5}-\frac{8}{109} \mathrm{~h}_{2,6}=\mathrm{F}_{2, \mathrm{z}}
\end{aligned}
$$

Projections on the node 3:

$$
\begin{aligned}
& \frac{6}{\sqrt{109}} \mathrm{~h}_{2,3}+\mathrm{h}_{3,4}-\frac{5}{\sqrt{114}} \mathrm{~h}_{3,7}+\frac{11}{\sqrt{210}} \mathrm{~h}_{3,8}=\mathrm{F}_{3, \mathrm{x}} \\
& \frac{6}{\sqrt{73}} \mathrm{~h}_{1,3}+\frac{6}{\sqrt{109}} \mathrm{~h}_{2,3}+\frac{5}{\sqrt{114}} \mathrm{~h}_{3,7}+\frac{5}{\sqrt{210}} \mathrm{~h}_{3,8}=\mathrm{F}_{3, \mathrm{y}} \\
& -\frac{8}{\sqrt{73}} \mathrm{~h}_{1,3}-\frac{8}{\sqrt{109}} \mathrm{~h}_{2,3}-\frac{8}{\sqrt{114}} \mathrm{~h}_{3,7}+\frac{8}{\sqrt{210}} \mathrm{~h}_{3,8}=\mathrm{F}_{3, \mathrm{z}}
\end{aligned}
$$

Projections on the node 4:

$$
\begin{aligned}
& -\frac{6}{\sqrt{109}} \mathrm{~h}_{1,4}-\mathrm{h}_{3,4}-\frac{11}{\sqrt{210}} \mathrm{~h}_{4,7}+\frac{5}{\sqrt{114}} \mathrm{~h}_{4,8}=\mathrm{F}_{4, \mathrm{x}} \\
& \frac{3}{\sqrt{109}} \mathrm{~h}_{1,4}+\frac{3}{\sqrt{73}} \mathrm{~h}_{2,4}-\mathrm{h}_{4,5}+\frac{5}{\sqrt{210}} \mathrm{~h}_{4,7}+\frac{5}{\sqrt{114}} \mathrm{~h}_{4,8}=\mathrm{F}_{4, \mathrm{y}} \\
& -\frac{8}{\sqrt{109}} \mathrm{~h}_{1,4}-\frac{8}{\sqrt{73}} \mathrm{~h}_{2,4}-\frac{8}{\sqrt{210}} \mathrm{~h}_{4,7}-\frac{4}{\sqrt{114}} \mathrm{~h}_{4,8}=\mathrm{F}_{4, \mathrm{z}}
\end{aligned}
$$

Projections on the node 5:

$$
\begin{aligned}
& -\frac{6}{\sqrt{109}} h_{1,5}-h_{5,6}+\frac{5}{\sqrt{210}} h_{5,8}+\frac{5}{\sqrt{114}} h_{5,9}-\frac{11}{\sqrt{210}} h_{5,10}=F_{5, x} \\
& -\frac{3}{\sqrt{109}} h_{1,5}-\frac{3}{\sqrt{73}} h_{2,5}-h_{4,5}+\frac{11}{\sqrt{210}} h_{5,8}-\frac{5}{\sqrt{114}} h_{5,9}-\frac{5}{\sqrt{210}} h_{5,10}=F_{5, y} \\
& -\frac{8}{\sqrt{109}} h_{1,5}-\frac{8}{\sqrt{73}} h_{2,4}-\frac{8}{\sqrt{210}} h_{5,8}-\frac{8}{\sqrt{114}} h_{5,9}-\frac{8}{\sqrt{210}} h_{5,10}=F_{5, z}
\end{aligned}
$$

Projections on the node 6:

$$
-\frac{6}{\sqrt{109}} \mathrm{~h}_{2,6}-\mathrm{h}_{5,6}-\frac{5}{\sqrt{210}} \mathrm{~h}_{6,7}+\frac{11}{\sqrt{210}} \mathrm{~h}_{6,9}-\frac{5}{\sqrt{114}} \mathrm{~h}_{6,10}=\mathrm{F}_{6, \mathrm{x}}
$$


International Journal of Mathematical, Engineering and Management Sciences

Vol. 4, No. 1, 1-16, 2019

https://dx.doi.org/10.33889/IJMEMS.2019.4.1-001

$-\frac{3}{73} h_{1,6}-\frac{3}{\sqrt{109}} h_{2,6}-h_{3,6}+\frac{11}{\sqrt{210}} h_{6,7}-\frac{5}{\sqrt{210}} h_{6,9}-\frac{5}{\sqrt{114}} h_{6,10}=F_{6, y}$

$-\frac{4}{73} h_{1,6}-\frac{8}{\sqrt{109}} h_{2,6}-\frac{8}{\sqrt{210}} h_{6,7}-\frac{8}{\sqrt{210}} h_{6,9}-\frac{8}{\sqrt{114}} h_{6,10}=F_{6, z}$

\section{Formulation of the Structural Optimization Problem}

Size and shape optimization of truss structure is widely used despite large computation costs for massive structures. This optimization is often applied to obtain the optimum values of the cross section area $\mathrm{Si}, \mathrm{j}$ and lengths li which lead to minimum weight design. Our approach imposes the loading conditions and movements of nodes which include the calculations of cross sections and internal forces hi,j with the objective of mass minimization.

\subsection{Design Variables}

The variables that are chosen to describe the design of truss system are represented in Tables 5, 6 and 7.

Table 5. Variables $\left(x_{i}\right)_{1 \leq i \leq 25}$ of the cross sections $\left(S_{i, j}\right)_{1 \leq i, j \leq 25, i \neq j}$

\begin{tabular}{|c|c|}
\hline Cross Sections & Unknown variables \\
\hline $\mathrm{S}_{1, \mathrm{j}}$ & $\mathrm{x}_{1}$ \\
\hline $\mathrm{S}_{2, \mathrm{j}}$ & $\mathrm{x}_{2}$ \\
\hline$S_{3, j}$ & $\mathrm{x}_{3}$ \\
\hline $\mathrm{S}_{4, \mathrm{j}}$ & $\mathrm{x}_{4}$ \\
\hline $\mathrm{S}_{5, \mathrm{j}}$ & $\mathrm{x}_{5}$ \\
\hline $\mathrm{S}_{6, \mathrm{j}}$ & $\mathrm{x}_{6}$ \\
\hline $\mathrm{S}_{7, \mathrm{j}}$ & $\mathrm{x}_{7}$ \\
\hline$S_{8, j}$ & $\mathrm{x}_{8}$ \\
\hline $\mathrm{S}_{9, \mathrm{j}}$ & $\mathrm{X}_{9}$ \\
\hline $\mathrm{S}_{10, \mathrm{j}}$ & $\mathrm{x}_{10}$ \\
\hline$S_{11, j}$ & $\mathrm{x}_{11}$ \\
\hline$S_{12, \mathrm{j}}$ & $\mathrm{x}_{12}$ \\
\hline$S_{13, j}$ & $\mathrm{x}_{13}$ \\
\hline$S_{14, j}$ & $\mathrm{x}_{14}$ \\
\hline $\mathrm{S}_{15, \mathrm{j}}$ & $\mathrm{x}_{15}$ \\
\hline$S_{16, j}$ & $\mathrm{x}_{16}$ \\
\hline $\mathrm{S}_{17, \mathrm{j}}$ & $x_{17}$ \\
\hline $\mathrm{S}_{18, \mathrm{j}}$ & $\mathrm{x}_{18}$ \\
\hline $\mathrm{S}_{19, \mathrm{j}}$ & $\mathrm{x}_{19}$ \\
\hline$S_{20, j}$ & $\mathrm{x}_{20}$ \\
\hline$S_{21, j}$ & $\mathrm{x}_{21}$ \\
\hline$S_{22, j}$ & $\mathrm{x}_{22}$ \\
\hline $\mathrm{S}_{23, \mathrm{j}}$ & $\mathrm{x}_{23}$ \\
\hline$S_{24, j}$ & $\mathrm{x}_{24}$ \\
\hline$S_{25, j}$ & $\mathrm{X}_{25}$ \\
\hline
\end{tabular}


International Journal of Mathematical, Engineering and Management Sciences

Vol. 4, No. 1, 1-16, 2019

https://dx.doi.org/10.33889/IJMEMS.2019.4.1-001

Table 6.Variables $\left(\mathrm{x}_{\mathrm{i}}\right)_{25 \leq \mathrm{i} \leq 50}$ of the Internal Forcesh $_{\mathrm{i}, \mathrm{j}}$

\begin{tabular}{|c|c|}
\hline Internal Forces & Unknown variables \\
\hline $\mathrm{h}_{1,2}$ & $\mathrm{X}_{26}$ \\
\hline $\mathrm{h}_{1,3}$ & $\mathrm{X}_{27}$ \\
\hline $\mathrm{h}_{1,4}$ & $\mathrm{X}_{28}$ \\
\hline $\mathrm{h}_{1,5}$ & $\mathrm{X}_{29}$ \\
\hline $\mathrm{h}_{1,6}$ & $\mathrm{X}_{30}$ \\
\hline $\mathrm{h}_{2,3}$ & $\mathrm{X}_{31}$ \\
\hline $\mathrm{h}_{2,4}$ & $\mathrm{X}_{32}$ \\
\hline $\mathrm{h}_{2,5}$ & $\mathrm{X}_{33}$ \\
\hline $\mathrm{h}_{2,6}$ & $\mathrm{X}_{34}$ \\
\hline $\mathrm{h}_{3,4}$ & $\mathrm{X}_{35}$ \\
\hline $\mathrm{h}_{3,6}$ & $\mathrm{X}_{36}$ \\
\hline $\mathrm{h}_{3,7}$ & $\mathrm{X}_{37}$ \\
\hline $\mathrm{h}_{3,8}$ & $\mathrm{X}_{38}$ \\
\hline $\mathrm{h}_{3,10}$ & $\mathrm{X}_{39}$ \\
\hline $\mathrm{h}_{4,5}$ & $\mathrm{x}_{40}$ \\
\hline $\mathrm{h}_{4,7}$ & $\mathrm{x}_{41}$ \\
\hline $\mathrm{h}_{4,8}$ & $\mathrm{X}_{42}$ \\
\hline $\mathrm{h}_{4,9}$ & $\mathrm{X}_{43}$ \\
\hline $\mathrm{h}_{5,6}$ & $\mathrm{X}_{44}$ \\
\hline $\mathrm{h}_{5,8}$ & $\mathrm{X}_{45}$ \\
\hline $\mathrm{h}_{5,9}$ & $\mathrm{X}_{46}$ \\
\hline $\mathrm{h}_{5,10}$ & $\mathrm{X}_{47}$ \\
\hline $\mathrm{h}_{6,7}$ & $\mathrm{X}_{48}$ \\
\hline $\mathrm{h}_{6,9}$ & $\mathrm{X}_{49}$ \\
\hline $\mathrm{h}_{6,10}$ & $\mathrm{X}_{50}$ \\
\hline
\end{tabular}

Table 7. Variables $\left(\mathrm{x}_{\mathrm{i}}\right)_{25 \leq \mathrm{i} \leq 50}$ of Material Density $\rho_{\mathrm{i}}$

\begin{tabular}{|c|c|}
\hline Material Density & Unknown variables $s$ \\
\hline$\rho_{1}$ & $\mathrm{x}_{51}$ \\
\hline$\rho_{2}$ & $\mathrm{x}_{52}$ \\
\hline$\rho_{3}$ & $\mathrm{X}_{53}$ \\
\hline$\rho_{4}$ & $\mathrm{X}_{54}$ \\
\hline$\rho_{5}$ & $\mathrm{X}_{55}$ \\
\hline$\rho_{6}$ & $\mathrm{x}_{56}$ \\
\hline$\rho_{7}$ & $\mathrm{x}_{57}$ \\
\hline$\rho_{8}$ & $\mathrm{X}_{58}$ \\
\hline$\rho_{9}$ & $\mathrm{X}_{59}$ \\
\hline$\rho_{10}$ & $\mathrm{x}_{60}$ \\
\hline$\rho_{11}$ & $\mathrm{x}_{61}$ \\
\hline$\rho_{12}$ & $\mathrm{x}_{62}$ \\
\hline$\rho_{13}$ & $\mathrm{x}_{63}$ \\
\hline$\rho_{14}$ & $\mathrm{x}_{64}$ \\
\hline$\rho_{15}$ & $\mathrm{x}_{65}$ \\
\hline$\rho_{16}$ & $\mathrm{x}_{66}$ \\
\hline$\rho_{17}$ & $\mathrm{x}_{67}$ \\
\hline$\rho_{18}$ & $\mathrm{x}_{68}$ \\
\hline$\rho_{19}$ & $\mathrm{x}_{69}$ \\
\hline$\rho_{20}$ & $\mathrm{x}_{70}$ \\
\hline$\rho_{21}$ & $\mathrm{x}_{71}$ \\
\hline$\rho_{22}$ & $\mathrm{x}_{72}$ \\
\hline$\rho_{23}$ & $\mathrm{x}_{73}$ \\
\hline$\rho_{24}$ & $\mathrm{x}_{74}$ \\
\hline$\rho_{25}$ & $\mathrm{x}_{75}$ \\
\hline
\end{tabular}

\subsection{Linear Design Constraints}

The limitations of the truss system are behavior and side constraints in matrix form $A_{e q} x=b_{e q}$ and $A_{\text {ineq }} \mathrm{X} \leq \mathrm{b}_{\text {ineq }}$.

\subsubsection{Equality Constraints}

The first design requirement for $\mathrm{A}_{\mathrm{eq}} \in \mathrm{IR}^{19 \times 75}, \mathrm{~b}_{\mathrm{eq}} \in \mathrm{IR}^{19 \times 1}$ and $\mathrm{x} \in \mathrm{IR}^{75 \times 1}$ are represented by the equality constrains expressed by: 
International Journal of Mathematical, Engineering and Management Sciences

Vol. 4, No. 1, 1-16, 2019

https://dx.doi.org/10.33889/IJMEMS.2019.4.1-001

$$
\begin{aligned}
& \mathrm{x}_{26}+\frac{6}{\sqrt{109}} \mathrm{x}_{28}+\frac{6}{\sqrt{109}} \mathrm{x}_{29}=\mathrm{F}_{1, \mathrm{x}} \\
& \frac{3}{\sqrt{73}} \mathrm{x}_{27}+\frac{3}{\sqrt{109}} \mathrm{x}_{28}-\frac{3}{\sqrt{109}} \mathrm{x}_{29}-\frac{3}{73} \mathrm{x}_{30}=\mathrm{F}_{1, \mathrm{y}} \\
& -\frac{8}{\sqrt{73}} \mathrm{x}_{27}-\frac{8}{\sqrt{109}} \mathrm{x}_{28}-\frac{8}{\sqrt{109}} \mathrm{x}_{29}-\frac{8}{73} \mathrm{x}_{30}=\mathrm{F}_{1, \mathrm{z}} \\
& \mathrm{x}_{26}-\frac{6}{\sqrt{109}} \mathrm{x}_{31}-\frac{6}{\sqrt{109}} \mathrm{x}_{34}=\mathrm{F}_{2, \mathrm{x}} \\
& \frac{3}{\sqrt{109}} \mathrm{x}_{31}+\frac{3}{\sqrt{73}} \mathrm{x}_{32}-\frac{3}{\sqrt{73}} \mathrm{x}_{33}-\frac{3}{109} \mathrm{x}_{34}=\mathrm{F}_{2, \mathrm{y}} \\
& -\frac{8}{\sqrt{109}} \mathrm{x}_{31}-\frac{8}{\sqrt{73}} \mathrm{x}_{32}+\frac{8}{\sqrt{73}} \mathrm{x}_{33}-\frac{8}{109} \mathrm{x}_{34}=\mathrm{F}_{2, \mathrm{z}} \\
& \frac{6}{\sqrt{109}} \mathrm{x}_{31}+\mathrm{x}_{35}-\frac{5}{\sqrt{114}} \mathrm{x}_{37}+\frac{11}{\sqrt{210}} \mathrm{x}_{38}=\mathrm{F}_{3, \mathrm{x}} \\
& \frac{6}{\sqrt{73}} \mathrm{x}_{27}+\frac{6}{\sqrt{109}} \mathrm{x}_{31}+\frac{5}{\sqrt{114}} \mathrm{x}_{37}+\frac{5}{\sqrt{210}} \mathrm{x}_{38}=\mathrm{F}_{3, \mathrm{y}} \\
& -\frac{8}{\sqrt{73}} \mathrm{x}_{27}-\frac{8}{\sqrt{109}} \mathrm{x}_{31}-\frac{8}{\sqrt{114}} \mathrm{x}_{37}+\frac{8}{\sqrt{210}} \mathrm{x}_{38}=\mathrm{F}_{3, \mathrm{z}} \\
& -\frac{6}{\sqrt{109}} \mathrm{x}_{28}-\mathrm{x}_{35}-\frac{11}{\sqrt{210}} \mathrm{x}_{41}+\frac{5}{\sqrt{114}} \mathrm{x}_{42}=\mathrm{F}_{4, \mathrm{x}} \\
& \frac{3}{\sqrt{109}} \mathrm{x}_{28}+\frac{3}{\sqrt{73}} \mathrm{x}_{32}-\mathrm{x}_{40}+\frac{5}{\sqrt{210}} \mathrm{x}_{41}+\frac{5}{\sqrt{114}} \mathrm{x}_{42}=\mathrm{F}_{4, \mathrm{y}} \\
& -\frac{8}{\sqrt{109}} \mathrm{x}_{28}-\frac{8}{\sqrt{73}} \mathrm{x}_{32}-\frac{8}{\sqrt{210}} \mathrm{x}_{41}-\frac{4}{\sqrt{114}} \mathrm{x}_{42}=\mathrm{F}_{4, \mathrm{z}} \\
& -\frac{6}{\sqrt{109}} \mathrm{x}_{29}-\mathrm{x}_{44}+\frac{5}{\sqrt{210}} \mathrm{x}_{45}+\frac{5}{\sqrt{114}} \mathrm{x}_{46}-\frac{11}{\sqrt{210}} \mathrm{x}_{47}=\mathrm{F}_{5, \mathrm{x}} \\
& -\frac{3}{\sqrt{109}} \mathrm{x}_{29}-\frac{3}{\sqrt{73}} \mathrm{x}_{33}-\mathrm{x}_{40}+\frac{11}{\sqrt{210}} \mathrm{x}_{45}-\frac{5}{\sqrt{114}} \mathrm{x}_{46}-\frac{5}{\sqrt{210}} \mathrm{x}_{47}=\mathrm{F}_{5, \mathrm{y}} \\
& -\frac{8}{\sqrt{109}} \mathrm{x}_{29}-\frac{8}{\sqrt{73}} \mathrm{x}_{32}-\frac{8}{\sqrt{210}} \mathrm{x}_{45}-\frac{8}{\sqrt{210}} \mathrm{x}_{46}-\frac{8}{\sqrt{210}} \mathrm{x}_{47}=\mathrm{F}_{5, \mathrm{z}} \\
& -\frac{8}{\sqrt{109}} \mathrm{x}_{29}-\frac{8}{\sqrt{73}} \mathrm{x}_{32}-\frac{8}{\sqrt{210}} \mathrm{x}_{45}-\frac{8}{\sqrt{210}} \mathrm{x}_{46}-\frac{8}{\sqrt{210}} \mathrm{x}_{47}=\mathrm{F}_{5, \mathrm{z}} \\
& -\frac{6}{\sqrt{109}} \mathrm{x}_{34}-\mathrm{x}_{44}-\frac{5}{\sqrt{210}} \mathrm{x}_{48}+\frac{11}{\sqrt{210}} \mathrm{x}_{49}-\frac{5}{\sqrt{114}} \mathrm{x}_{50}=\mathrm{F}_{6, \mathrm{x}} \\
& -\frac{3}{\sqrt{73}} \mathrm{x}_{30}-\frac{3}{\sqrt{109}} \mathrm{x}_{34}-\mathrm{x}_{36}+\frac{11}{\sqrt{210}} \mathrm{x}_{48}-\frac{5}{\sqrt{210}} \mathrm{x}_{49}-\frac{5}{\sqrt{114}} \mathrm{x}_{50}=\mathrm{F}_{6, \mathrm{y}} \\
& -\frac{4}{\sqrt{73}} \mathrm{x}_{30}-\frac{8}{\sqrt{109}} \mathrm{x}_{34}-\frac{8}{\sqrt{210}} \mathrm{x}_{48}-\frac{8}{\sqrt{210}} \mathrm{x}_{49}-\frac{8}{\sqrt{114}} \mathrm{x}_{50}=\mathrm{F}_{6, \mathrm{z}}
\end{aligned}
$$

An additional equality constraint on limited material density is given by:

$\sum_{\mathrm{i}=51}^{75} \mathrm{x}_{\mathrm{i}}=1925.25$

\subsubsection{Inequality Constraints}

The second design requirement for $A_{\text {ineq }} \in I R^{118 \times 75}, b_{\text {ineq }} \in I R^{118}$ and $x \in I R^{75}$ is represented by the inequality constraints expressed by:

$\left|\mathrm{x}_{26}\right| \leq \mathrm{x}_{1} \sigma_{0}, \quad\left|\mathrm{x}_{27}\right| \leq \mathrm{x}_{8} \sigma_{0}, \quad\left|\mathrm{x}_{28}\right| \leq \mathrm{x}_{2} \sigma_{0}$

$\left|\mathrm{x}_{29}\right| \leq \mathrm{x}_{4} \sigma_{0}, \quad\left|\mathrm{x}_{30}\right| \leq \mathrm{x}_{9} \sigma_{0}, \quad\left|\mathrm{x}_{31}\right| \leq \mathrm{x}_{3} \sigma_{0}$

$\left|\mathrm{x}_{32}\right| \leq \mathrm{x}_{6} \sigma_{0}, \quad\left|\mathrm{x}_{33}\right| \leq \mathrm{x}_{7} \sigma_{0}, \quad\left|\mathrm{x}_{34}\right| \leq \mathrm{x}_{5} \sigma_{0}$

$\left|\mathrm{x}_{35}\right| \leq \mathrm{x}_{12} \sigma_{0}, \quad\left|\mathrm{x}_{36}\right| \leq \mathrm{x}_{10} \sigma_{0}, \quad\left|\mathrm{x}_{37}\right| \leq \mathrm{x}_{19} \sigma_{0}$

$\left|\mathrm{x}_{38}\right| \leq \mathrm{x}_{23} \sigma_{0}, \quad\left|\mathrm{x}_{39}\right| \leq \mathrm{x}_{14} \sigma_{0}, \quad\left|\mathrm{x}_{40}\right| \leq \mathrm{x}_{11} \sigma_{0}$ 
International Journal of Mathematical, Engineering and Management Sciences

Vol. 4, No. 1, 1-16, 2019

https://dx.doi.org/10.33889/IJMEMS.2019.4.1-001

$\left|\mathrm{x}_{41}\right| \leq \mathrm{x}_{18} \sigma_{0}, \quad\left|\mathrm{x}_{42}\right| \leq \mathrm{x}_{24} \sigma_{0}, \quad\left|\mathrm{x}_{43}\right| \leq \mathrm{x}_{16} \sigma_{0}$

$\left|\mathrm{x}_{44}\right| \leq \mathrm{x}_{13} \sigma_{0}, \quad\left|\mathrm{x}_{45}\right| \leq \mathrm{x}_{17} \sigma_{0}, \quad\left|\mathrm{x}_{46}\right| \leq \mathrm{x}_{25} \sigma_{0}$

$\left|\mathrm{x}_{47}\right| \leq \mathrm{x}_{20} \sigma_{0}, \quad\left|\mathrm{x}_{48}\right| \leq \mathrm{x}_{15} \sigma_{0}, \quad\left|\mathrm{x}_{49}\right| \leq \mathrm{x}_{21} \sigma_{0}$

$\left|\mathrm{x}_{50}\right| \leq \mathrm{x}_{22} \sigma_{0}, \quad\left|\mathrm{x}_{26}\right| \leq \frac{2}{3} \mathrm{Eu}_{0}, \quad\left|\mathrm{x}_{27}\right| \leq \frac{26}{73} \mathrm{Eu}_{0}$

$\left|\mathrm{x}_{28}\right| \leq \frac{10}{209} \mathrm{Eu}_{0}, \quad\left|\mathrm{x}_{29}\right| \leq \frac{10}{209} \mathrm{Eu}_{0}, \quad\left|\mathrm{x}_{30}\right| \leq \frac{26}{73} \mathrm{Eu}_{0}$

$\left|\mathrm{x}_{31}\right| \leq \frac{10}{209} \mathrm{Eu}_{0}, \quad\left|\mathrm{x}_{32}\right| \leq \frac{26}{73} \mathrm{Eu}_{0}, \quad\left|\mathrm{x}_{33}\right| \leq \frac{26}{73} \mathrm{Eu}_{0}$

$\left|\mathrm{x}_{34}\right| \leq \frac{10}{209} \mathrm{Eu}_{0}, \quad 0 \leq\left(\mathrm{x}_{\mathrm{i}}\right)_{1 \leq \mathrm{i} \leq 25}, \quad 0 \leq\left(\mathrm{x}_{\mathrm{i}}\right)_{51 \leq \mathrm{i} \leq 75}$

\subsection{Nonlinear Objective Function}

One represents the structural mass of the truss system by the nonlinear objective function of several variables stated as:

$$
\begin{aligned}
\mathrm{m}(\mathrm{x})= & \sum_{\mathrm{i}=1}^{\mathrm{n}} \rho_{\mathrm{i}} \mathrm{s}_{\mathrm{i}} \mathrm{L}_{\mathrm{i}}=+\frac{\sqrt{109}}{2}\left(\mathrm{x}_{56} \mathrm{x}_{2}+\mathrm{x}_{57} \mathrm{x}_{3}+\mathrm{x}_{58} \mathrm{x}_{4}+\mathrm{x}_{59} \mathrm{x}_{5}\right) \\
& +\frac{\sqrt{73}}{2}\left(\mathrm{x}_{60} \mathrm{x}_{6}+\mathrm{x}_{61} \mathrm{x}_{7}+\mathrm{x}_{62} \mathrm{x}_{8}+\mathrm{x}_{63} \mathrm{x}_{9}\right) \\
+ & \frac{\sqrt{114}}{2}\left(\mathrm{x}_{72} \mathrm{x}_{22}+\mathrm{x}_{73} \mathrm{x}_{23}+\mathrm{x}_{74} \mathrm{x}_{24}+\mathrm{x}_{75} \mathrm{x}_{25}\right) \\
& +\frac{\sqrt{210}}{2}\left(\mathrm{x}_{64} \mathrm{x}_{14}+\mathrm{x}_{65} \mathrm{x}_{15}+\mathrm{x}_{66} \mathrm{x}_{16}+\mathrm{x}_{67} \mathrm{x}_{17}\right) \\
& +\frac{\sqrt{210}}{2}\left(\mathrm{x}_{68} \mathrm{x}_{18}+\mathrm{x}_{69} \mathrm{x}_{19}+\mathrm{x}_{70} \mathrm{x}_{20}+\mathrm{x}_{71} \mathrm{x}_{21}\right) \\
& +3\left(\mathrm{x}_{51} \mathrm{x}_{1}+\mathrm{x}_{52} \mathrm{x}_{10}+\mathrm{x}_{53} \mathrm{x}_{11}+\mathrm{x}_{54} \mathrm{x}_{12}+\mathrm{x}_{55} \mathrm{x}_{13}\right)
\end{aligned}
$$

where $\mathrm{L}_{\mathrm{i}}, \rho_{\mathrm{i}}, \mathrm{S}_{\mathrm{i}}$ and $\mathrm{n}=25$ are respectively the length, material density, the cross-section area of the $\mathrm{i}^{\text {th }}$ element and the number of elements in the structure.

\subsection{Optimization Statement}

The structural optimization problem consists of finding the material density, stresses and cross section area which minimize the objective function $\mathrm{m}(\mathrm{x})$ under equality (41-60) and inequality (61-72) constraints. This can be stated as:

$$
\begin{aligned}
& \min _{x \in I R^{75}} m(x) \\
& \text { subject to }\left\{\begin{array}{c}
A_{e q} x=b_{\text {eq }} \\
A_{\text {ineq }} x \leq b_{\text {ineq }} \\
x, \text { unrestricted }
\end{array}\right.
\end{aligned}
$$

The interior point method is applied through the solver of type fmincom to solve the nonlinear minimization problem (74-75) using Matlab Optimization Toolbox (Coleman et al., 1999). 
International Journal of Mathematical, Engineering and Management Sciences

Vol. 4, No. 1, 1-16, 2019

https://dx.doi.org/10.33889/IJMEMS.2019.4.1-001

\section{Applications of the Structural Analysis}

\subsection{Additional Data}

The loading cases imposed for this structural analysis is given by the next Table 8 .

Table 8. Imposed loading conditions

\begin{tabular}{|c|c|c|c|c|}
\hline Loading cases & Nodes & Imposed load on $\mathbf{x}$-axis $(\mathbf{k N})$ & Imposed load on y-axis (kN) & $\begin{array}{c}\text { Imposed load on z-axis } \\
(\mathbf{k N})\end{array}$ \\
\hline 1 & 1 & 100 & 100 & -50 \\
\hline 1 & 2 & 0 & 100 & -50 \\
\hline 1 & 3 & 50 & 0 & 0 \\
\hline 2 & 5 & 0 & 200 & -50 \\
\hline 2 & 6 & 0 & -200 & 50 \\
\hline
\end{tabular}

We also have the following parameters:

- Coefficient $\mathrm{C}_{\alpha}=0.04$

- Partial coefficient of security $\gamma_{\mathrm{MO}}=1.1$

- Limit of elasticity $\mathrm{f}_{\mathrm{y}}=235 \mathrm{MPa}$

- Stress limit in compression or tension $\sigma_{0}=\frac{\mathrm{f}_{\mathrm{y}}}{\gamma_{\mathrm{MO}}}=213.63 \mathrm{MPa}$

- Minimum cross section area $S_{\min }=1.0 \mathrm{~cm}^{2}$.

We define CSA, VIF, SN, and MD respectively as the cross-section area, values of internal forces, stress nature and material density.

\subsection{Result of the Loading Case 1}

Through the loading case 1 , the minimal value of the structural mass $m_{1}=25679.755 \mathrm{~kg}$ is found at the fifth iteration and the decisions parameters that provide this mass are presented in Table 9.

In Table 9, we find that the material density is the same at each cross section and it values 7.701 $\mathrm{kg} / \mathrm{m}^{3}$. The internal force vanishes where the cross-section areas $\mathrm{x}_{10}, \mathrm{x}_{14}$ and $\mathrm{x}_{18}$ are not equal to zero. This result shows that the internal forces are inactive at the cross section areas $\mathrm{x}_{10}, \mathrm{x}_{14}$ and $\mathrm{x}_{18}$. We observe that there are 13 elements in compression and only 9 in tension in the results of the loading case 1 . 
International Journal of Mathematical, Engineering and Management Sciences

Vol. 4, No. 1, 1-16, 2019

https://dx.doi.org/10.33889/IJMEMS.2019.4.1-001

Table 9. Optimal values of cross-sectional area $\left(x_{i}\right)_{1 \leq i \leq 25}$ for the loading case 1

\begin{tabular}{|c|c|c|c|}
\hline $\operatorname{CSA}\left(\mathrm{cm}^{2}\right)$ & VIF (kN) & SN & $\mathbf{M D}\left(\mathrm{kg} / \mathrm{m}^{3}\right)$ \\
\hline $\mathrm{x}_{1}=2.2389$ & $x_{26}=-47.830$ & Compression & 7.701 \\
\hline $\mathrm{x}_{2}=10.5525$ & $x_{27}=-15.393$ & Compression & 7.701 \\
\hline$x_{3}=2.7220$ & $\mathrm{x}_{28}=225.440$ & Tension & 7.701 \\
\hline $\mathrm{x}_{4}=1.4881$ & $\mathrm{x}_{29}=31.792$ & Tension & 7.701 \\
\hline $\mathrm{x}_{5}=6.6177$ & $\mathrm{x}_{30}=-141.717$ & Compession & 7.701 \\
\hline $\mathrm{x}_{6}=171.4113$ & $\mathrm{x}_{31}=58.151$ & Tension & 7.701 \\
\hline $\mathrm{x}_{7}=177.10944$ & $\mathrm{x}_{32}=-41.534$ & Compression & 7.701 \\
\hline $\mathrm{x}_{8}=170.4755$ & $\mathrm{x}_{33}=-163.045$ & Compression & 7.701 \\
\hline $\mathrm{x}_{9}=176.1110$ & $x_{34}=-141.379$ & Compression & 7.701 \\
\hline $\mathrm{x}_{10}=2.3419$ & $\mathrm{x}_{35}=0.000$ & - & 7.701 \\
\hline $\mathrm{x}_{11}=3.9908$ & $\mathrm{x}_{36}=50.031$ & Tension & 7.701 \\
\hline $\mathrm{x}_{12}=1.0000$ & $x_{37}=-44.258$ & Compression & 7.701 \\
\hline $\mathrm{x}_{13}=1.000$ & $x_{38}=-5.460$ & Compression & 7.701 \\
\hline $\mathrm{x}_{14}=1.0000$ & $\mathrm{x}_{39}=0.000$ & - & 7.701 \\
\hline $\mathrm{x}_{15}=2.9177$ & $x_{40}=-85.259$ & Compression & 7.701 \\
\hline $\mathrm{x}_{16}=1.0000$ & $\mathrm{x}_{41}=-92.355$ & Compression & 7.701 \\
\hline $\mathrm{x}_{17}=1.6548$ & $\mathrm{x}_{42}=-221.207$ & Compression & 7.701 \\
\hline $\mathrm{x}_{18}=4.3230$ & $\mathrm{x}_{43}=0.000$ & - & 7.701 \\
\hline $\mathrm{x}_{19}=1.0000$ & $\mathrm{x}_{44}=13.489$ & Tension & 7.701 \\
\hline $\mathrm{x}_{20}=1.0000$ & $\mathrm{x}_{45}=-35.353$ & Compression & 7.701 \\
\hline $\mathrm{x}_{21}=1.7557$ & $\mathrm{x}_{46}=60.572$ & Tension & 7.701 \\
\hline $\mathrm{x}_{22}=7.4694$ & $x_{47}=-20.541$ & Compression & 7.701 \\
\hline $\mathrm{x}_{23}=1.0000$ & $\mathrm{x}_{48}=62.332$ & Tension & 7.701 \\
\hline $\mathrm{x}_{24}=10.3543$ & $\mathrm{x}_{49}=37.509$ & Tension & 7.701 \\
\hline $\mathrm{x}_{25}=2.8353$ & $\mathrm{x}_{50}=159.573$ & Tension & 7.701 \\
\hline
\end{tabular}

\subsection{Result of the Loading Case 2}

The minimal value of the structural mass $\mathrm{m}_{2}=1963.908 \mathrm{~kg}$ is found at the second iteration and the decisions parameters that provide this mass are presented in the Table 10. Through the loading case 2, we have also the material density equal to $7.701 \mathrm{~kg} / \mathrm{m}^{3}$. The same observation was done in loading case 1 is made with loading case 2, namely, the internal forces are inactive at the cross section areas $\mathrm{x}_{9}, \mathrm{x}_{14}$ and $\mathrm{x}_{18}$. These results show that the structural mass corresponding to the worst loading is case 1 with $\mathrm{m}_{1}=25679.755 \mathrm{~kg}$ compared to case 2 with lesser effects on the structure. 
International Journal of Mathematical, Engineering and Management Sciences

Vol. 4, No. 1, 1-16, 2019

https://dx.doi.org/10.33889/IJMEMS.2019.4.1-001

Table 10. Optimal values of cross-sectional area $\left(\mathrm{x}_{\mathrm{i}}\right)_{1 \leq \mathrm{i} \leq 25}$ for the loading case 2

\begin{tabular}{|c|c|c|c|}
\hline $\operatorname{CSA}\left(\mathrm{cm}^{2}\right)$ & VIF (kN) & SN & MD $\left(\mathrm{kg} / \mathrm{m}^{3}\right)$ \\
\hline $\mathrm{x}_{1}=1.0000$ & $x_{26}=-5.081$ & Compression & 7.701 \\
\hline $\mathrm{x}_{2}=1.0000$ & $\mathrm{x}_{27}=-1.208$ & Compression & 7.701 \\
\hline $\mathrm{x}_{3}=1.0000$ & $\mathrm{x}_{28}=1.476$ & Tension & 7.701 \\
\hline $\mathrm{x}_{4}=1.0000$ & $\mathrm{x}_{29}=7.365$ & Tension & 7.701 \\
\hline $\mathrm{x}_{5}=1.0000$ & $\mathrm{x}_{30}=-6.027$ & Compession & 7.701 \\
\hline $\mathrm{x}_{6}=1.0000$ & $\mathrm{x}_{31}=-8.840$ & Compression & 7.701 \\
\hline $\mathrm{x}_{7}=1.0000$ & $x_{32}=-2.326$ & Compression & 7.701 \\
\hline $\mathrm{x}_{8}=1.0000$ & $x_{33}=-9.560$ & Compression & 7.701 \\
\hline $\mathrm{x}_{9}=1.0000$ & $\mathrm{x}_{34}=0.000$ & - & 7.701 \\
\hline $\mathrm{x}_{10}=5.0905$ & $\mathrm{x}_{35}=9.428$ & Tension & 7.701 \\
\hline $\mathrm{x}_{11}=1.0000$ & $\mathrm{x}_{36}=108.753$ & Tension & 7.701 \\
\hline $\mathrm{x}_{12}=1.0000$ & $\mathrm{x}_{37}=11.432$ & Tension & 7.701 \\
\hline $\mathrm{x}_{13}=1.8753$ & $\mathrm{x}_{38}=1.432$ & Tension & 7.701 \\
\hline $\mathrm{x}_{14}=1.0000$ & $\mathrm{x}_{39}=0.000$ & - & 7.701 \\
\hline $\mathrm{x}_{15}=5.2082$ & $\mathrm{x}_{40}=-1.470$ & Compression & 7.701 \\
\hline $\mathrm{x}_{16}=1.0000$ & $\mathrm{x}_{41}=6.915$ & Compression & 7.701 \\
\hline$x_{17}=9.6594$ & $\mathrm{X}_{42}=-7.396$ & Compression & 7.701 \\
\hline $\mathrm{x}_{18}=1.0000$ & $\mathrm{x}_{43}=0.000$ & - & 7.701 \\
\hline $\mathrm{x}_{19}=1.0000$ & $\mathrm{x}_{44}=40.065$ & Tension & 7.701 \\
\hline $\mathrm{x}_{20}=1.0000$ & $\mathrm{x}_{45}=206.360$ & Tension & 7.701 \\
\hline $\mathrm{x}_{21}=1.0000$ & $\mathrm{x}_{46}=-79.786$ & Compression & 7.701 \\
\hline $\mathrm{x}_{22}=1.0000$ & $\mathrm{x}_{47}=-13.778$ & Compression & 7.701 \\
\hline $\mathrm{x}_{23}=1.0000$ & $\mathrm{x}_{48}=-111.226$ & Compression & 7.701 \\
\hline $\mathrm{x}_{24}=1.0000$ & $\mathrm{x}_{49}=9.580$ & Tension & 7.701 \\
\hline $\mathrm{x}_{25}=3.7346$ & $\mathrm{x}_{50}=11.955$ & Tension & 7.701 \\
\hline
\end{tabular}

\section{Conclusion}

In this paper, the behavior of a twenty-five bars spatial truss has been described by a linear elastic model with imposed loading conditions acting on five node elements. The unique solution of the elastic model is found by minimizing the energy functional in the solution space of all the displacements and then used to formulate the mass minimization problem as a nonlinear minimization problem. For the two loading cases, the results of this structural optimization show the characteristics of the cross section areas, material densities and internal forces which satisfy the effectiveness of the minimum mass design. It appears that the minimum mass obtained with the loading case 2 is less than the mass obtained with loading case 1 . We have found that the best structural design corresponding to our experimental data is the one which has an economical mass, uses less financial resources and allows substantial changes in size, shape, and topology. 
International Journal of Mathematical, Engineering and Management Sciences

Vol. 4, No. 1, 1-16, 2019

https://dx.doi.org/10.33889/IJMEMS.2019.4.1-001

\section{Conflict of Interest}

The authors confirm that there is no conflict of interest to declare for this publication.

\section{Acknowledgement}

This manuscript would not have been possible without the cooperation between the Departments of Mathematics and Civil Engineering at the National Advanced School of Engineering of the University of Yaounde I (NASE/ UYI) in Cameroon.

We would also like to thank the African Center of Excellence in Information and Communication Technologies of the University of Yaounde I for their collaboration and support.

\section{References}

Adeli, H., \& Kamal (1986). Efficient optimization of space trusses. Computers \& Structures, 24(3), 501511.

Adeli, H., \& Park, H. S. (1996). Hybrid CPN-neural dynamics model for discrete optimization of steel structures. Computer-Aided Civil and Infrastructure Engineering, 11(5), 355-366.

Bora, A. (2016). Comparative study of tubular steel truss profiles for roofing varying span. International Journal of Engineering Research \& Technology, 5(4), 495-499.

Coleman, T., Branch, A. M., \& Grace, A. (1999). Optimization Toolbox for Use with Matlab (Version 2).The MathWorks, Inc.

Erbatur, F., Hasançebi, O., Tütüncü, I., \& Hilic, H. (2000). Optimal design of planar and space structures with genetic algorithms. Computers \& Structures, 75(2), 209-224.

Farshi, B., \& Ziazi, A. (2010). Sizing optimization of truss structures by methode of centers and force formulation. International Journal of Solids and Structures, 47(18-19), 2508-2524.

Herskovits, J., Dias, G., Santos, G., \& Mota Soares, C. M. (2000). Shape structural optimization with an interior point nonlinear programming algorithm. Structural and Multidisciplinary Optimization, 20(2), $107-115$.

Jha, C. K., \& Paliwal, M. C. (2017). Fully stressed design of Howe truss using STAAD.PRO software. International Journal of Engineering Development and Research, 5(3), 1127-1131.

Lamberti, L. (2008). An efficient simulated annealing algorithm for design optimization of truss structures. Computers \& Structures, 86(19-20), 1936-1953.

Park, H. S., \& Sung, C. W. (2002). Optimization of steel structures using distributed simulated annealing algorithm on a cluster of personal computers. Computers \& Structures, 80(14-15), 1305-1316.

Patrikar, A., \& Pathak, K. K. (2016). Fully stressed design of Fink truss using STAAD.Pro software. Open Journal of Civil Engineering, 6(4), 631-642.

Patvegar, S. M., \& Datye, V. P. (2016). Comparative parametric study of Vierendeel girder and truss girder for single track railway bridge. International Research Journal of Engineering and Technology, 3(6), 3066-3070.

Rajeev, S., \& Krishnamoorthy, C. S. (1992). Discrete optimization of structures using genetic algorithms. Journal of Structural Engineering, 118(5), 1233-1250. 
International Journal of Mathematical, Engineering and Management Sciences

Vol. 4, No. 1, 1-16, 2019

https://dx.doi.org/10.33889/IJMEMS.2019.4.1-001

Saka, M. P. (1990). Optimum design of pin-jointed steel structures with practical applications. Journal Structural Engineering, 116(10), 2599-2620.

Schmit, L. A., \& Farshi, B. (1974). Some approximation concepts for structural synthesis. AIAA Journal, 12(5), 692-699.

Venkayya, V. B. (1971). Design of optimum structures. Computers \& Structures, 1(1-2), 265-309.

Wu, S. J., \& Chow, P. T. (1995a). Integrated discrete and configuration optimization of trusses using genetic algorithms. Computers \& Structures, 55(4), 695-702.

Wu, S. J., \& Chow, P. T. (1995b). Steady-state genetic algorithms for discrete optimization of trusses. Computer and Structures, 56(6), 979-991.

\section{Websites}

https://ntrs.nasa.gov/archive/nasa/casi.ntrs.nasa.gov/19760011449.pdf

Schmit, L. A., \& Miura, H. (1976). Approximation concepts for efficient structural synthesis. Retrieved from National Aeronautics and Space AdministrationWebsite. 